\section{COMPETÊNCIAS ESSENCIAIS DESENVOLVIDAS POR COORDENADORES DE CENTROS DE SAÚDE DA FAMÍLIA}

\author{
Core competencies developed by managers of family health \\ centers
}

\author{
Competencias esenciales desarrolladas por coordinadores de \\ centros de salud de la familia
}

\section{RESUMO}

Objetivo: Descrever o perfil dos coordenadores dos Centros de Saúde da Família (CSF) de Fortaleza-CE e a frequência do desenvolvimento das competências essenciais na gerência de seus serviços. Método: Estudo quantitativo, descritivo e analítico, realizado nos CSF de Fortaleza-CE, em 2012, com amostra composta por 30 CSF do município. Entrevistaramse os coordenadores dos CSF e seus supervisores, além dos chefes de Distrito de Saúde (DS) das Secretarias Executivas Regionais (SER). Aplicou-se um questionário junto aos coordenadores referente a perfil epidemiológico, experiências profissionais anteriores, formação acadêmica e caracterização do vínculo empregatício. Por meio de uma escala, identificaram-se as competências e atividades desenvolvidas pela gerência: habilidades e atribuições. Aos chefes de DS, aplicou-se um recorte do questionário, e o teste Kappa identificou a concordância entre a frequência do relatado pelos chefes e os coordenadores. Resultados: Encontrou-se o perfil dos coordenadores formado por mulheres $(n=25 ; 83 \%)$, com mais de 45 anos ( $n=19 ; 63,3 \%)$, graduadas em Enfermagem $(n=12 ; 40 \%)$ e especialistas $(\mathrm{n}=18 ; 60 \%)$, com experiência anterior em área administrativa $(n=20 ; 66,7 \%)$. As competências mais frequentes foram: tecnologias leves $(\mathrm{n}=20 ; 63,3 \%)$, gerência de materiais $(n=24 ; 80 \%)$, aceitar diferenças $(n=25 ; 83,3 \%)$ e trabalho em equipe $(n=25 ; 83,3 \%)$. Não houve concordância $(\mathrm{k}=-0,418 ; \mathrm{p}=0,014<0,05)$ entre chefes e coordenadores. Conclusão: $\mathrm{O}$ perfil de coordenadores foi representado por mulheres com experiência anterior em atividades administrativas, capacitadas por meio de cursos de pós-graduação. Identificouse alta frequência no desenvolvimento da maioria das competências essenciais, porém, a avaliação dos chefes de DS esteve abaixo do esperado.

Descritores: Avaliação de Serviços de Saúde; Atenção Primária à Saúde; Administração de Serviços de Saúde.

\section{ABSTRACT}

Objective: To describe the profile of Family Health Center (FHC) managers in Fortaleza, Ceara, Brazil, and the frequency of core competencies development in the management of their services. Method: Quantitative, descriptive and analytical study performed in Fortaleza, Ceara, in 2012, with sample consisting of 30 FHC. The FHC managers and the chiefs of Health Districts (HD) of the Regional Executive Secretariats (RES) were interviewed. The questionnaire applied to the managers regarded the epidemiological profile, previous professional experience, academic background, and characterization of employment relationship. Through a scale, the competences and activities developed by the managers were identified: skills and attributions. The HD chiefs answered a questionnaire clipping, and the Kappa test identified the correlation between the frequency of what was reported by the managers and the chiefs. Results: The managers' profile was composed by a majority of women $(n=25 ; 83 \%)$, above 45 years of age $(n=19 ; 63.3 \%)$, nursing graduate $(n=12 ; 40 \%)$ and postgraduate $(n=18 ; 60 \%)$, with previous experience in the administrative area $(n=20 ; 66.7 \%)$. The most common competencies were: light technologies $(n=20$; $63.3 \%)$, materials management $(n=24 ; 80 \%)$, accepting differences $(n=25 ; 83.3 \%)$ and
Artigo Original

Nayane Coelho Sales ${ }^{(1)}$

Marcelo Gurgel Carlos da Silva ${ }^{(1)}$ Francisco José Maia Pinto ${ }^{(1)}$

1) Universidade Estadual do Ceará - UECE - Fortaleza (CE) - Brasil
Recebido em: 30/04/2013 Revisado em: $15 / 07 / 2013$ Aceito em: 18/02/2014 
teamwork $(n=25 ; 83.3 \%)$. There was not agreement $(k=-0.418$; $p=0.014<0.05)$ between chiefs and managers. Conclusion: The managers' profile was represented by women with previous experience in the administrative area, qualified by postgraduate programs. A high frequency in the development of the majority of the core competencies was identified, although the assessment of the chiefs has resulted below expectations.

Descriptors: Health Services Evaluation, Primary Health Care, Health Services Management.

\section{RESUMEN}

Objetivo: Describir el perfil de los coordinadores de los Centros de Salud de la Familia (CSF) de Fortaleza-CE y la frecuencia del desarrollo de las competencias esenciales para la gerencia de sus servicios. Métodos: Estudio cuantitativo, descriptivo y analítico realizado en los CSF de Fortaleza-CE, en 2012, con una muestra de 30 CSF del municipio. Se entrevistó a los coordinadores de los CSF y sus supervisores y los jefes de Distrito de Salud (DS) de las Secretarias Ejecutivas Regionales (SER). Se aplicó un cuestionario a los coordinadores sobre el perfil epidemiológico, las experiencias profesionales previas, la formación académica y la caracterización del vínculo de empleo. A través de una escala se identificaron las competencias y actividades desarrolladas por la gerencia: habilidades e atribuciones. Se aplicó un recorte del cuestionario a los jefes de DS y la prueba de Kappa identificó la concordancia entre la frecuencia de lo que fue relatado por los jefes y coordinadores. Resultados: El perfil de los coordinadores fue de mujeres $(n=25 ; 83 \%)$ con más de 45 años ( $n=19 ; 63,3 \%)$, enfermeras $(n=12 ; 40 \%)$, especialistas $(n=18 ; 60 \%)$ y con experiencia previa en el área administrativo $(n=20 ; 66,7 \%)$. Las competencias más frecuentes fueron: tecnologías leves ( $n=20$; $63,3 \%)$, gerencia de materiales $(n=24 ; 80 \%)$, aceptación de las diferencias $(n=25 ; 83,3 \%)$ y trabajo en equipo $(n=25 ; 83,3 \%)$. No hubo concordancia $(k=-0,418 ; p=0,014<0,05)$ entre los jefes y coordinadores. Conclusión: El perfil de los coordinadores fue representado por mujeres con experiencia previa en actividades administrativas, capacitadas a través de cursos de pos-grado. Se identificó frecuencia elevada en el desarrollo de la mayoría de las competencias esenciales, sin embargo, la evaluación de los jefes de DS estuvo abajo del esperado.

Descriptores: Evaluación de Servicios de Salud; Atención Primaria de Salud; Administración de los Servicios de Salud.

\section{INTRODUÇÃO}

A Atenção Primária em Saúde (APS) é considerada internacionalmente como a base para um novo modelo assistencial de sistemas de saúde que tenham em seu centro o usuário. Refere-se a um conjunto de práticas integrais em saúde direcionadas a responder necessidades individuais e coletivas. No Brasil, durante o processo de implementação do Sistema Único de Saúde (SUS), passou a ser denominada Atenção Básica à Saúde $(\mathrm{ABS})^{(1)}$.

Fazendo parte de seus princípios norteadores, a promoção da saúde é um olhar renovado sobre a saúde, com participação social, ação intersetorial, fortalecimento das capacidades individuais e coletivas, definição de políticas públicas e reorientação dos serviços de saúde ${ }^{(2)}$. Como campo aplicado de conhecimentos e práticas, a promoção da saúde tem nas abordagens avaliativas um instrumento importante de legitimação do seu caráter inovador ${ }^{(3)}$.

Tão complexo quanto esse sistema é a sua gestão, pois nela se encontram múltiplos fatores implicados. Essa tarefa exige bem mais que vontade política e senso comum; requer uma sólida base de conhecimentos. Porém, o que se percebe, muitas vezes, é o uso de improvisação e empirismo ${ }^{(4)}$.

Ator fundamental nesse processo de mudanças é o gestor local, que, na "ponta" do sistema, costuma ser denominado gerente, por sua função administrativa e gerencial ${ }^{(5)}$. Para exercer essa função complexa, é preciso ter conhecimento das áreas da saúde e da administração, ter uma visão geral do contexto em que se está inserido, compromisso social com a comunidade ${ }^{(6)}$, além de um conhecimento sólido dos fundamentos que alicerçam os sistemas de serviços de saúde ${ }^{(7)}$. Devem conhecer globalmente os serviços desempenhados no local de trabalho, pois as competências requeridas implicam o conceito transversal da integração dos conhecimentos, saberes técnicos, metodológicos e das relações intersubjetivas ${ }^{(8)}$.

Alcançar esses objetivos na gerência dos serviços de APS é uma tarefa complexa para a maioria dos gestores que estão à frente das unidades de saúde. Historicamente, a gerência era apenas executora das ações planejadas no âmbito federal, não acumulando experiências em planejar, desenvolver e avaliar políticas de saúde. O processo de descentralização do SUS colocou a competência gerencial como um fator preocupante para a implementação de um sistema regionalizado, hierarquizado e participativo ${ }^{(9)}$.

Além de adequada capacitação técnica, o perfil de um bom gerente compreende liderança, motivação, comunicação, capacidade para lidar com conflitos e ética. $\mathrm{O}$ perfil gerencial pode, então, ser definido como um conjunto de atitudes, aptidões e habilidades mediante as quais o gerente desenvolve a gestão dos serviços de saúde ${ }^{(10)}$.

Essa tríade foi primeiramente citada no estudo intitulado The Core Competence of the Corporation ${ }^{(11)}$. As competências essenciais da gerência que devem estar presentes na organização de uma empresa podem ser dividas entre atitudes, conhecimentos e habilidades. Uma competência essencial pode ser representada como uma função multiplicadora de fatores, por exemplo: variadas tecnologias (duras e leves), aprendizado coletivo (multinível 
e multifuncional) e capacidade de compartilhar (além das fronteiras empresariais e geográficas). Afirma-se que, para desenvolvê-las, são necessárias a educação continuada dos funcionários, o uso contínuo das competências e a aplicação destas no trabalho em equipe ${ }^{(12)}$.

O presente estudo procura, portanto, descrever o perfil dos coordenadores dos Centros de Saúde da Família (CSF) de Fortaleza-CE e a frequência do desenvolvimento das competências essenciais na gerência de seus serviços.

\section{MÉTODOS}

Trata-se de um estudo do tipo quantitativo, com caráter descritivo e analítico, realizado em Fortaleza-CE, entre os meses de maio e dezembro de 2012, com amostra composta por 30 do total de 91 CSF do município.

Utilizou-se o tamanho mínimo de uma amostra - 30 (trinta) - para a realização de cálculos estatísticos ${ }^{(13)}$. Esse valor corresponde a $33 \%$ do total da população pesquisada, obtendo-se, assim, amostra não probabilística para o presente estudo.

O critério de inclusão compreendeu unidades com coordenadores em exercício da função no local pesquisado há no mínimo um ano, sendo excluídos aqueles que não estavam exercendo suas atividades no período da coleta de dados por motivo de licença ou afastamento.

Distribuiu-se a amostra proporcionalmente entre as seis Secretarias Executivas Regionais (SER), unidades administrativas do município, de acordo com a quantidade de unidades adstritas a cada uma das regionais. Realizou-se a seleção das unidades por meio de sorteio, por pesquisador não integrante da equipe de coleta de dados. A substituição das unidades, quando necessária, também se deu por sorteio.

O primeiro instrumento era um questionário aplicado junto aos coordenadores dos CSF, contendo perguntas referentes a perfil epidemiológico, experiências profissionais anteriores, formação acadêmica e caracterização do vínculo empregatício com a Prefeitura de Fortaleza. Através de uma escala de Likert adaptada ${ }^{(14)}$, buscou-se identificar as competências e atividades desenvolvidas pela gerência. Essa escala é composta por trinta perguntas, agrupadas em três categorias: habilidades, atribuições e competências. As respostas da escala de seis pontos variam entre: sempre, quase sempre, frequentemente, raramente, nunca e sem resposta, com atribuições de valores para cada uma de 5, 4, $3,2,1,0$, respectivamente. Solicitou-se aos entrevistados que indicassem com que frequência exerciam as atividades e competências na administração dos seus serviços.

O segundo questionário foi um recorte do primeiro, aplicado aos chefes dos Distritos de Saúde (DS) de cada SER. Contém seis das 30 perguntas do primeiro, com duas questões de cada uma das três categorias, sobre as competências essenciais desenvolvidas pelos coordenadores sob sua supervisão. As perguntas foram as de número $11,15,19,21,25$ e 34 , versando respectivamente sobre planejamento, negociação, análise das condições de saúde, demanda espontânea, trabalho em equipe e correção de desempenho.

Construiu-se o banco de dados em planilhas, exportadas posteriormente para o Statistical Package for the Social Sciences (SPSS), versão 17, no qual se realizou análise descritiva e inferencial. Por meio de tabelas, apresenta-se a análise descritiva com frequências absolutas e relativas. Para análise inferencial entre as respostas dos coordenadores e chefes de DS, construiu-se uma escala com os valores atribuídos às frequências, considerando-se a concentração dos escores à direita em uma ordem crescente, utilizando como ponto de corte o valor 4. Valores maiores ou iguais a esse ponto foram considerados satisfatórios ou altos e valores menores foram considerados baixos ou insatisfatórios.

Posteriormente, o teste de Kappa identificou o grau de concordância entre as respostas dos coordenadores e as respostas dos chefes dos DS. Essa concordância varia de +1 a -1 , em que +1 representa concordância total e -1 significa o oposto. Todos os testes foram realizados com nível de significância de 0,05 .

A inserção dos participantes no estudo se deu após assinatura de Termo de Consentimento Livre e Esclarecido, em duas vias, conforme orientação da Resolução 466/12 do Conselho Nacional de Saúde ${ }^{(15)}$. A coleta de dados ocorreu com avaliação e aprovação no Comitê de Ética em Pesquisa da Universidade Estadual do Ceará (Parecer $n^{\circ}$ 26934/2012), autorização da Comissão de Pesquisa da Secretaria Municipal de Saúde Escola de Fortaleza (SMSE) e consentimento de cada chefe de Distrito das regionais de saúde.

\section{RESULTADOS}

A Tabela I apresenta o perfil dos coordenadores dos CSF entrevistados. A faixa etária de grande parte dos coordenadores $(n=19 ; 63,3 \%)$ foi de 45 anos ou mais, com variação de 28 a 58 anos de idade. A maioria $(n=25 ; 83 \%)$ era do sexo feminino.

A formação básica dos coordenadores era a graduação na área da Assistência em Saúde, com apenas um deles com curso superior em Administração Hospitalar. O bacharelado em Enfermagem corresponde à maioria $(\mathrm{n}=12 ; 40 \%)$. As demais profissões aparecem em menor número, com dois cirurgiões-dentistas, dois fisioterapeutas e um profissional das áreas de Terapia Ocupacional, Medicina e Nutrição. 
Tabela I - Perfil e formação coordenadores dos Centros de Saúde da Família. Fortaleza-CE, 2013.

\begin{tabular}{|c|c|}
\hline Variável & n (\%) \\
\hline \multicolumn{2}{|l|}{ Idade (em anos) } \\
\hline $25-34$ & $1(3,3)$ \\
\hline $35-44$ & $10(30,3)$ \\
\hline$\geq 45$ & $19(63,3)$ \\
\hline \multicolumn{2}{|l|}{ Sexo } \\
\hline Feminino & $25(83,0)$ \\
\hline Masculino & $5(17,0)$ \\
\hline \multicolumn{2}{|l|}{ Formação acadêmica } \\
\hline Graduação & $9(30,0)$ \\
\hline Especialização & $18(60,0)$ \\
\hline Mestrado & $3(10,0)$ \\
\hline \multicolumn{2}{|c|}{ Experiência profissional anterior(') } \\
\hline Administrativa & $20(66,7)$ \\
\hline Assistência Hospitalar & $10(30,3)$ \\
\hline Ensino & $2(6,7)$ \\
\hline PSF & $3(10,0)$ \\
\hline \multicolumn{2}{|c|}{ Vínculo empregatício com a prefeitura $\left({ }^{2}\right)$} \\
\hline Servidor público & $11(36,7)$ \\
\hline Terceirizado & $4(13,3)$ \\
\hline Comissionado & $9(30,0)$ \\
\hline \multicolumn{2}{|c|}{ Carga horária semanal na unidade } \\
\hline $20 \mathrm{~h}$ & $1(3,3)$ \\
\hline $40 \mathrm{~h}$ & $29(96,7)$ \\
\hline
\end{tabular}

(1) Cada profissional podia responder a mais de uma área em que tinha experiência; (2) Seis profissionais não responderam a essa pergunta e não foi possível obter tal informação de outra fonte.

A especialização representou o nível de pós-graduação mais frequente, com $18(60 \%)$ especialistas, não sendo possível determinar o curso de maior prevalência, pois poucos entrevistados fizeram essa especificação nos instrumentos. A maioria $(\mathrm{n}=20 ; 66,7 \%)$ referiu experiência anterior na área administrativa.
A segunda parte do primeiro instrumento corresponde à frequência com que os coordenadores realizavam as atividades e competências essenciais em suas unidades, descrita nas Tabelas II, III e IV.

$\mathrm{O}$ item que obteve maior frequência da resposta "sempre" na categoria Habilidades foi o Uso de Tecnologia

Tabela II - Frequência da categoria Habilidades dos Coordenadores dos Centros de Saúde da Família. Fortaleza-CE, 2013.

\begin{tabular}{|c|c|c|c|c|c|c|c|c|c|c|c|c|c|c|}
\hline \multirow{2}{*}{ Frequência } & \multicolumn{2}{|c|}{ Comunicação } & \multicolumn{2}{|c|}{ Organização } & \multicolumn{2}{|c|}{ Planejamento } & \multicolumn{2}{|c|}{$\begin{array}{c}\text { Tomada de } \\
\text { Decisão }\end{array}$} & \multicolumn{2}{|c|}{ Tecn. Leve* } & \multicolumn{2}{|c|}{ Tecn. Dura* } & \multicolumn{2}{|c|}{ Negociação } \\
\hline & $\mathrm{n}$ & $\%$ & $\mathrm{n}$ & $\%$ & $\mathrm{n}$ & $\%$ & n & $\%$ & $\mathrm{n}$ & $\%$ & $\mathrm{n}$ & $\%$ & $\mathrm{n}$ & $\%$ \\
\hline Sem Resposta & - & - & - & - & - & - & - & - & 1 & 3,3 & - & - & 3 & 10,0 \\
\hline Nun & - & - & - & - & - & - & - & - & - & - & - & - & - & - \\
\hline Rar & - & - & - & - & - & - & - & - & - & - & 1 & 3.3 & - & - \\
\hline mente & 2 & 6,7 & 3 & 10,0 & 5 & $167-2 \cdot 0 \cdot 0$ & 3 & 10,0 & 3 & 10,0 & 3 & 10 & 5 & 16,7 \\
\hline Quase Sempre & 9 & 30,0 & 8 & 26 & 9 & 30,0 & 8 & 26,7 & 6 & 20,0 & 10 & 33 & 3 & 10,0 \\
\hline Sempre & 19 & 63,3 & 19 & 63,3 & 16 & 53,3 & 19 & 63,3 & 20 & 66,7 & 16 & 53,3 & 19 & 63,3 \\
\hline
\end{tabular}

*Tecn. Leve.: tecnologia leve; Tecn. Dura: tecnologia dura 
Tabela III - Frequência da categoria Atribuições dos Coordenadores dos Centros de Saúde da Família. Fortaleza-CE, 2013.

\begin{tabular}{|c|c|c|c|c|c|c|c|c|c|c|c|c|c|c|c|c|}
\hline \multirow[t]{2}{*}{ Frequência } & \multicolumn{2}{|c|}{ Autonomia } & \multicolumn{2}{|c|}{$\begin{array}{c}\text { Articulação } \\
\text { Governo }\end{array}$} & \multicolumn{2}{|c|}{$\begin{array}{c}\text { Articulação } \\
\text { ONG* }\end{array}$} & \multicolumn{2}{|c|}{$\begin{array}{l}\text { Análise Cond. } \\
\text { Saúde* } \\
\end{array}$} & \multicolumn{2}{|c|}{$\begin{array}{l}\text { Vig. San./ } \\
\text { Epidem.* }\end{array}$} & \multicolumn{2}{|c|}{$\begin{array}{c}\text { Ações } \\
\text { Program.* }\end{array}$} & \multicolumn{2}{|c|}{$\begin{array}{l}\text { Demanda } \\
\text { Espontânea }\end{array}$} & \multicolumn{2}{|c|}{$\begin{array}{r}\text { Gerência } \\
\text { Materiais }\end{array}$} \\
\hline & $\mathbf{n}$ & $\%$ & $\mathrm{n}$ & $\%$ & $\bar{n}$ & $\%$ & $\mathrm{n}$ & $\%$ & $\mathbf{n}$ & $\%$ & $\mathrm{n}$ & $\%$ & $\mathbf{n}$ & $\%$ & $\mathrm{n}$ & $\%$ \\
\hline Sem Resposta & 1 & 3,3 & - & - & - & - & - & - & - & - & - & - & - & - & 1 & 3,3 \\
\hline Nunca & - & ? & - & - & 2 & 6,7 & 1 & 3,3 & - & - & 1 & 3,3 & - & - & - & - \\
\hline Raramente & 1 & 3,3 & 6 & 20,0 & 3 & 10,0 & - & - & 1 & 3,3 & 1 & 3,3 & - & - & - & - \\
\hline Frequentemente & 6 & 20,0 & 4 & 13,3 & 7 & 23,3 & 3 & 10,0 & 2 & 6,7 & 3 & 10,0 & 3 & 10,0 & 1 & 3,3 \\
\hline Quase Sempre & 12 & 40,0 & 7 & 23,3 & 6 & 20,0 & 11 & 36,7 & 8 & 26,7 & 5 & 16,7 & 8 & 26,7 & 4 & 13,3 \\
\hline Sempre & 10 & 33,3 & 13 & 43,3 & 12 & 40,0 & 15 & 50,0 & 19 & 63,3 & 20 & 66,7 & 19 & 63,3 & 24 & 80,0 \\
\hline
\end{tabular}

* ONG: Organização Não Governamental; Cond.: Condições de Saúde; Vig. San. Epidem.: Vigilância Sanitária Epidemiológica; Program.: Programáticas.

Leve, com 20 (66,7\%) gestores. Essa é uma característica marcante nos modelos de saúde da Atenção Primária. Um pouco menos frequente: Comunicação, Organização, Tomada de Decisão e Negociação, todos com 19 (63,3\%) respostas "sempre".
$\mathrm{Na}$ categoria Atribuições, a maior prevalência encontrada $(\mathrm{n}=24 ; 80,0 \%)$ ficou na Gerência de Materiais. A maioria dos itens apareceu com altos escores, porém, destacou-se negativamente a atribuição Autonomia, com apenas $10(33,3 \%)$ respostas "sempre".

Tabela IV - Frequência da categoria Competências dos Coordenadores dos Centros de Saúde da Família. Fortaleza-CE, 2013.

\begin{tabular}{|c|c|c|c|c|c|c|c|c|c|c|c|c|c|c|c|c|}
\hline \multicolumn{17}{|c|}{ Parte A } \\
\hline \multirow[t]{2}{*}{ Frequência } & \multicolumn{2}{|c|}{ Liderança } & \multicolumn{2}{|c|}{$\begin{array}{l}\text { Gerência } \\
\text { Conflitos }\end{array}$} & \multicolumn{2}{|c|}{$\begin{array}{l}\text { Trabalho } \\
\text { em Equipe }\end{array}$} & \multicolumn{2}{|c|}{$\begin{array}{c}\text { Aceita } \\
\text { Diferenças }\end{array}$} & \multicolumn{2}{|c|}{ Resiliência } & \multicolumn{2}{|c|}{$\begin{array}{l}\text { Acomoda } \\
\text { Mudanças }\end{array}$} & \multicolumn{2}{|c|}{$\begin{array}{l}\text { Estímulo/ } \\
\text { Motivação }\end{array}$} & \multicolumn{2}{|c|}{$\begin{array}{c}\text { Segue } \\
\text { Procedimentos }\end{array}$} \\
\hline & $\mathbf{n}$ & $\%$ & $\mathbf{n}$ & $\%$ & $\mathrm{n}$ & $\%$ & $\mathbf{n}$ & $\%$ & $\mathbf{n}$ & $\%$ & $\mathbf{n}$ & $\%$ & $\mathbf{n}$ & $\%$ & $\mathbf{n}$ & $\%$ \\
\hline Sem & - & - & - & - & 1 & 3,3 & 1 & 3,3 & - & - & - & - & - & - & - & - \\
\hline Nunc & - & - & - & - & - & - & - & - & - & 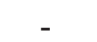 & 1 & 3, & 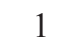 & 3,3 & 1 & 3,3 \\
\hline Raramen & - & - & 1 & 3,3 & - & - & 1 & 3,3 & - & - & 2 & 6,7 & - & - & 4 & 13,3 \\
\hline Frequentemente & 3 & 10,0 & 1 & 3,3 & - & - & 2 & 6,7 & 4 & 13,3 & 2 & 6,7 & 2 & 6,7 & 4 & 13,3 \\
\hline Quase sempre & 3 & 10,0 & 5 & 16,7 & 4 & 13,3 & 1 & 3,3 & 15 & 50,0 & 7 & 23,3 & 5 & 16,7 & 1 & 3,3 \\
\hline Sempre & 24 & 80,0 & 23 & 76,7 & 25 & 83,3 & 25 & 83,3 & 11 & 36,7 & 18 & 60,0 & 22 & 73,3 & 20 & 66,7 \\
\hline
\end{tabular}

\section{Parte B}

\begin{tabular}{|c|c|c|c|c|c|c|c|c|c|c|c|c|c|c|}
\hline \multirow[t]{2}{*}{ Frequência } & \multicolumn{2}{|c|}{$\begin{array}{c}\text { Preenchimento } \\
\text { Relatórios }\end{array}$} & \multicolumn{2}{|c|}{$\begin{array}{l}\text { Alocação } \\
\text { Materiais }\end{array}$} & \multicolumn{2}{|c|}{$\begin{array}{l}\text { Alocação Rec. } \\
\text { Human.* }\end{array}$} & \multicolumn{2}{|c|}{$\begin{array}{c}\text { Corrige } \\
\text { Desempenho }\end{array}$} & \multicolumn{2}{|c|}{$\begin{array}{c}\text { Auxilia } \\
\text { Aprendizado }\end{array}$} & \multicolumn{2}{|c|}{$\begin{array}{c}\text { Operação } \\
\text { Computadores }\end{array}$} & \multicolumn{2}{|c|}{$\begin{array}{c}\text { Previne } \\
\text { Problemas }\end{array}$} \\
\hline & $\mathbf{n}$ & $\%$ & $\mathbf{n}$ & $\%$ & n & $\%$ & $\mathbf{n}$ & $\%$ & $n$ & $\%$ & $\mathbf{n}$ & $\%$ & $\mathbf{n}$ & \\
\hline Sem Resposta & 2 & 6,7 & 1 & 3,3 & 2 & 6,7 & 2 & 6,7 & & 3, & 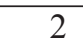 & 6,7 & 3 & 10,0 \\
\hline Nunc & - & 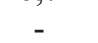 & - & - & - & - & - & 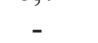 & 2 & 6 & 1 & 3,3 & 1 & 3,3 \\
\hline Raramen & - & - & - & - & - & - & 1 & 3,3 & 1 & 3,3 & 1 & 3,3 & 1 & 3,3 \\
\hline Frequentemente & 2 & 6,7 & 3 & 10,0 & - & - & 4 & 13,3 & 3 & 10,0 & 3 & 10,0 & 5 & 16,7 \\
\hline Quase sempre & 3 & 10,0 & 8 & 26,7 & 7 & 23,3 & 7 & 23,3 & 8 & 26,7 & 9 & 30,0 & 10 & 33,3 \\
\hline Sempre & 23 & 76,7 & 18 & 60,0 & 21 & 70,0 & 16 & 53,3 & 15 & 50,0 & 14 & 46,7 & 10 & 33,3 \\
\hline
\end{tabular}

*Rec. Human.: Recursos Humanos

Os itens da categoria Competências obtiveram altos valores em sua maioria. Abaixo de $50 \%$, têm-se as questões relacionadas à operação de computadores $(n=14 ; 46,7 \%)$ e à prevenção de problemas com essas máquinas $(n=10$; $33,3 \%)$. Observou-se grande contraste entre os altos valores atribuídos aos itens Gerência de Conflitos ( $\mathrm{n}=23 ; 76,7 \%)$ e Aceitar Diferenças $(n=25 ; 83,3 \%)$, e o valor baixo de Resiliência ( $\mathrm{n}=11 ; 36,7 \%)$ para a resposta "sempre".

A Tabela V apresenta os resultados da aplicação do teste Kappa, calculado entre os escores dos coordenadores dos CSF e dos chefes de DS. 
Tabela V - Coeficiente Kappa calculado entre escores dos chefes de Distrito de Saúde e os coordenadores dos Centros de Saúde da Família. Fortaleza-CE, 2013.

\begin{tabular}{lcc}
\hline Ações desenvolvidas & $\begin{array}{c}\text { Coeficiente } \\
\text { kappa }\end{array}$ & $\boldsymbol{p}$ \\
\hline Planejamento & 0,204 & 0,261 \\
Negociação & 0,200 & 0,256 \\
Análise das condições de saúde & 0,000 & 1,000 \\
Demanda espontânea & $-0,133$ & 0,439 \\
Trabalho em equipe & $-0,418$ & 0,014 \\
Correção de desempenho & $-0,200$ & 0,232 \\
\hline
\end{tabular}

Apenas uma das seis perguntas do questionário, que versava sobre o trabalho em equipe, mostrou relação estatística significativa $(\mathrm{p}=0,014)$. Ainda assim, o valor do coeficiente foi muito pequeno e negativo $(k=-0,418)$, sugerindo discordância entre os dois grupos de avaliadores.

\section{DISCUSSÃO}

A categoria "profissional predominante na função de coordenador" no estudo em questão foi a de enfermeiro, comum em outras populações estudadas ${ }^{(16)}$.

O enfermeiro é um profissional que tem na sua formação uma maior ênfase na área administrativa do que a maioria das profissões na área de saúde. Nas diretrizes curriculares estabelecidas pelo Ministério da Educação (MEC), os profissionais enfermeiros devem estar aptos a tomar iniciativa e fazer gerenciamento e administração da força de trabalho, dos recursos físicos, materiais e de informação, da mesma forma que devem estar aptos a ser empreendedores, gestores, empregadores ou lideranças na equipe de saúde ${ }^{(17)}$.

A prevalência do sexo feminino no atual estudo se deve, em parte, pela maior presença de mulheres na enfermagem, que é uma profissão predominantemente feminina. Desde os tempos mais primórdios, essa profissão é exercida quase exclusivamente por mulheres. Em algumas culturas, cuidar dos doentes é considerado uma extensão das tarefas da mulher ${ }^{(18)}$.

A maior prevalência encontrada na presente pesquisa foi a faixa etária de mais de 45 anos, sugerindo que funções gerenciais demandam maior experiência profissional e idade, pois faz-se necessário um tempo prolongado para que se adquira um conjunto de conhecimentos e experiências sólidos na sua formação ${ }^{(19)}$.

O grande número de profissionais que realizou uma pósgraduação lato sensu indica uma maior preocupação em se capacitar e atualizar seus conhecimentos. Os profissionais reconhecem a importância de participar de um curso de pósgraduação lato sensu como forma de contribuição para a qualificação profissional e enriquecimento do conhecimento adquirido na prática assistencial ${ }^{(20)}$.

Muitos dos coordenadores investigados no presente estudo referiram experiência anterior na área administrativa. Isso é benéfico para os serviços, pois sugere mais maturidade e preparação para as situações encontradas na prática do gestor, visto que uma boa bagagem de conhecimento, capacidade de análise, capacidade de ação, aprimoramento de práticas e determinação em alcançar resultados se tornam indispensáveis para o perfil gerencial( ${ }^{(21)}$.

Comunicação, organização e planejamento foram habilidades com alta prevalência na amostra investigada. Comunicação é definida como a troca de informações, fatos, ideias e significados. A maior interferência na comunicação ocorre por conta dos "ruídos" de interpretação, capazes de distorcer a mensagem durante o processo comunicativo. A competência comunicacional é essencial, visto que, para organizar, é indispensável comunicar-se, a fim de estabelecer metas, canalizar energias e identificar/solucionar problemas. Aprender a comunicar-se com eficácia é crucial para incrementar a eficiência de cada unidade de trabalho e da organização como um todo ${ }^{(22)}$.

Em outro estudo, percebeu-se que, para os coordenadores, a importância do planejamento na ação gerencial relaciona-se principalmente à facilidade no alcance das metas e objetivos, e ao controle e estímulo às atividades ${ }^{(23)}$.

Tomada de decisão apareceu também com números elevados na investigação em questão, processo que pode ser desenvolvido pelos gestores com maior qualidade se estes seguirem um método. Para tanto, a avaliação contínua dos serviços constitui um instrumento essencial de apoio à gestão, pela sua capacidade de melhorar a qualidade da tomada de decisão ${ }^{(24)}$.

As tecnologias leves foram relatadas como amplamente utilizadas, sendo entendidas como acolhimento, vínculo, autonomização e resolutividade. Essas formas de tecnologias são uma característica marcante na ESF, podendo ser entendidas tanto como saber como por seus desdobramentos materiais e não materiais na produção dos serviços de saúde. São classificadas em leves, leve-duras e duras. As tecnologias leves são as relações; as leve-duras são as dos saberes estruturados/teorias; e as duras são as dos recursos materiais ${ }^{(25)}$.

No contexto da atenção primária, a negociação é um instrumento fundamental de ajuste, gerador de consenso, capaz de sustentar e viabilizar propostas de ação conjunta $^{(26)}$. Interessante destacar que, no presente estudo, a atribuição menos frequente foi a autonomia, cuja falta dificulta sobremaneira o desenvolvimento das atividades e a execução de mudanças para melhoria nas unidades de saúde, falhando na negociação. 
A análise das condições de saúde com base em dados epidemiológicos e da vigilância sanitária foi citada como "quase sempre" ou "sempre" pela maioria dos coordenadores investigados no atual estudo. Isso deveria se refletir em um adequado planejamento de ações e num maior alcance das demandas da população adstrita em seu território de abrangência. Resultado semelhante foi encontrado em outro estudo ${ }^{(27)}$, que identificou $67 \%$ de unidades com modelo tradicional de atenção básica utilizando dados de produção e dados epidemiológicos para o planejamento das atividades, enquanto nas unidades que adotavam a ESF esse percentual subia para $80 \%$. O planejamento não existe sem a epidemiologia ${ }^{(28)}$.

A programação e controle das ações de demanda espontânea, como acolhimento, humanização da porta de entrada e atividades educativas, são ações consideradas como componentes das tecnologias leves e obtiveram frequências relatadas bem semelhantes na amostra analisada. A maioria dos coordenadores disse exercer sempre uma boa liderança, com adequada habilidade para gerenciamento de conflitos e trabalho em equipe, aceitando diferenças étnicas, sociais e de formação. Contudo, esse cenário torna-se contraditório quando uma pequena porcentagem citou ser resiliente. Liderança é o processo de conduzir as ações ou influenciar o comportamento e a mentalidade de outras pessoas ${ }^{(29)}$. É o processo pelo qual um grupo é induzido a dedicar-se aos objetivos defendidos pelo líder e seus seguidores. Liderança e administração se sobrepõem, já que alguns aspectos da liderança poderiam ser descritos como gerenciamento. Para que o líder possa administrar satisfatoriamente o conflito, é necessário entender o seu processo. $\mathrm{O}$ diálogo com a equipe, bem como o adequado manejo do trabalho do grupo, tornase ferramenta útil para gerenciar os conflitos, que devem ser vistos como oportunidade de crescimento ${ }^{(30)}$.

O preenchimento de relatórios e a alimentação dos sistemas de informação foram referidos como sempre realizadas na sua prática na atual investigação. Essas informações são de suma importância para o diagnóstico local em saúde e o planejamento das ações de saúde no âmbito da Unidade Básica de Saúde. O aumento exponencial dos gastos com saúde no mundo, nas duas últimas décadas, e as restrições orçamentárias representam grandes desafios para os sistemas universais de saúde ${ }^{(31)}$.

O domínio de técnicas de operação de tecnologias recentes, de máquinas e computadores, e a prevenção/ manutenção desses equipamentos mostraram-se com baixa frequência na amostra pesquisada. Isso pode ocorrer porque a população de coordenadores apresenta maior predomínio na faixa etária de mais de 45 anos, fazendo parte de uma geração que sofreu um contato mais tardio com essas tecnologias ${ }^{(32)}$.
Todavia, a presença das competências essenciais na prática dos coordenadores foi vista de outra forma pelos chefes dos Distritos de Saúde das SER, seus supervisores. Percebeu-se esse fato pela diferença na média dos escores e na classificação: a maioria das respostas dos chefes de Distrito mostrou uma frequência inferior àquela relatada pelos próprios coordenadores. O fenômeno observado de que a autoavaliação apresentou pontuações mais elevadas que a avaliação realizada por superiores hierárquicos pode ser devido a um receio de que os sujeitos pudessem ser prejudicados em seu local de trabalho ou a uma dificuldade na percepção das limitações de seu trabalho. Esse obstáculo foi percebido de forma semelhante na utilização da autoavaliação em outros estudos ${ }^{(33)}$.

Os resultados da aplicação do teste Kappa confirmam a análise descritiva dos dados do presente estudo. Apenas uma das seis perguntas realizadas mostrou relação estatística significativa, e ainda assim negativa, indicando discordância entre os avaliadores do mesmo fenômeno.

A partir da discussão do estudo, percebem-se algumas limitações, destacando-se as percepções diferentes de uma mesma realidade, pois o coordenador, ao realizar sua autoavaliação, possivelmente foi levado a fazê-la de forma positiva, por estar inserido no meio e temer represálias. Já os chefes de Distritos, por serem supervisores, podem ter direcionado sua posição de forma mais crítica e voltada para as deficiências de seus subordinados. A pesquisa não pôde aferir o grau de comprometimento dos coordenadores, bem como se a formação e capacitação foram específicas para as atividades de gerenciamento e entendimento dos princípios norteadores do sistema de saúde. Um maior enfoque nesses últimos aspectos pode trazer um maior retorno em resultados de qualidade para os serviços de saúde. Os dados apresentados no presente estudo, por si só, não representam a realidade do município, mas, através da discussão com aspectos da realidade local, fatos observados durante a coleta dos dados e as colocações realizadas por outros autores da literatura, podem-se levantar questões a serem repensadas e discutidas para melhorias da gestão do sistema.

\section{CONCLUSÃO}

$\mathrm{O}$ perfil de profissionais investigados à frente da coordenação dos Centros de Saúde da Família (CSF) de Fortaleza é representado por mulheres com experiência anterior em atividades administrativas e capacitadas por meio de cursos de pós-graduação.

A frequência das competências essenciais, sob a ótica dos coordenadores, foi alta na maioria dos itens, embora pontuadas com baixos valores questões como autonomia, 
domínio de tecnologias de informática e manutenção de equipamentos. Entretanto, na visão dos chefes de Distrito de Saúde, isso não se converteu em uma boa administração desses serviços, evidenciando a discordância de percepção.

\section{REFERÊNCIAS}

1 Giovanella L, Mendonça, MHM. Atenção Primária à Saúde. In: Giovanella L, Escorel S, Lobato LVC, Noronha JC, Carvalho AI. Políticas e Sistemas de Saúde no Brasil. Rio de Janeiro: Fiocruz; 2008. p. 575625.

2. Ministério da Saúde (BR). Promoção da saúde: Carta de Otawa, Declaração de Adelaide, Sundswall e Santa Fé de Bogotá. Brasília; 2001.

3. Lopes ALM, Silva AS, Castro DFA, Bógus CM, Fracoli LA. Avaliação de programas, serviços e tecnologias na perspectiva da promoção da saúde: uma reflexão teórica. Rev Bras Promoç Saúde. 2013;26(4):590-4.

4. Mamede S. Prefácio. In: Os Sistemas de Serviços de Saúde: o que os gestores deveriam saber sobre essas organizações complexas. Fortaleza: Escola de Saúde Pública do Ceará; 2002. p.13-14.

5. Ministério da Saúde (BR). Glossário do Ministério da Saúde: projeto de terminologia em saúde. Brasília: Ministério da Saúde; 2004.

6. Passos JP, Ciosak SI. A concepção dos enfermeiros no processo gerencial em Unidade Básica de Saúde. Rev Esc Enferm USP. 2006;40(4):464-8.

7. Mendes EV. Os sistemas de serviços de saúde: o que os gestores deveriam saber sobre essas organizações complexas. Fortaleza: Escola de Saúde Pública do Ceará; 2002.

8. Zarifian P. El modelo de competência y los sistemas productivos. Montevideo: Cinterfor; 1999.

9. Fernandes LCL, Machado RZ, Anschau GO. Gerência de serviços de saúde: Competências desenvolvidas e dificuldades encontradas na atenção básica. Ciênc Saúde Coletiva. 2009;14(Supl 1)1541-52.

10. Alves LAAR. Perfil gerencial do enfermeiro para atuar na atenção primária à saúde. In: Santos AS, Miranda MRC. A enfermagem na gestão em atenção primária à saúde. Barueri: Manole; 2007.

11. Prahalad CK, Hamel G. The Core Competence of the Corporation. Boston: Harvard Business Review; 1990.

12. Lazzarotto EM, Cardoso GM, Nazzari RK. Conhecimento, habilidades e atitudes requeridas para o enfermeiro gerenciar unidades básicas de saúde - UBS. In: Anais do Seminário Nacional Estado e Políticas Sociais; 26 a 28 Jun. 2003; Universidade Estadual do Oeste do Paraná (UNIOESTE); Núcleo de Estudos e Pesquisas em Políticas Sociais (NEPPS). Cascaval: Universidade Estadual do Oeste do Paraná (UNIOESTE); 2003. p. 1-15.

13. CochranWG. SamplingTechniques. New York: Nem York John Wiley; 1977.

14. Ávila DVB. O perfil do gestor de unidade básica de saúde: verificando competências e habilidades [monografia]. Porto Alegre: Universidade Estadual do Rio Grande do Sul, 2005.

15. Ministério da Saúde (BR), Conselho Nacional de Saúde. Resolução no 466 de 12 de Dezembro 2012. Diretrizes e Normas Regulamentadoras de Pesquisa envolvendo Seres Humanos. Brasília: Ministério da Saúde, 2012.

16. Ohira RHF, Cordoni Júnior L, Nunes EFPA. Perfil dos gerentes de Atenção Primária à Saúde de municípios de pequeno porte do norte do Paraná, Brasil. Ciênc Saúde Coletiva. 2014;19(2):393-400.

17. Ministério da Educação (BR), Conselho Nacional de Educação. Parecer $n^{\circ} 1133$ de 01 de Novembro de 2001. Diretrizes Curriculares Nacionais dos Cursos de Graduação em Enfermagem, Medicina e Nutrição. Brasília: Ministério da Educação; 2001.

18. Carvalho DR, Kalinke LP. Perfil do enfermeiro quanto à motivação profissional e suas necessidades de desenvolvimento. Rev Boletim de Enfermagem. 2008;2(1):82-95.

19. Silva CC, Nascimento EM, Avelino BC, Durso SO, Colauto RD. Perfil profissional requerido dos contadores na região metropolitana de Belo Horizonte. In: Anais do V Congresso Nacional de Administração e Ciências Contábeis, 2014 Out 16, Rio de Janeiro. Rio de Janeiro: Universidade Federal do Rio de Janeiro, 2014. p. 1-15.

20. Oliveira NA, Thofehrn MB, Cecagno D, Siqueira HCH, Porto AR. Especialização em Projetos assistenciais de Enfermagem: contribuições na prática profissionais dos egressos. Texto \& Contexto Enferm. 2009;18(4):697704.

21. Ramires EP, Lourenção LG, Santos MR. Gerenciamento em Unidades Básicas de Saúde: conhecendo experiências. Arq Ciênc Saúde. 2004;11(4):205-9.

22. Peres AM, Ciampone MHT. Gerência e competências gerais do enfermeiro. Texto \& Contexto Enferm. 2006;15(3):492-9. 
23. Fernandes MC, Barros AS, Silva LMS, Nóbrega MFB, Silva MRF, Torres RAM. Análise da atuação do enfermeiro na gerência de unidades básicas de saúde. Rev Bras Enferm. 2010;63(1):11-5.

24. Tanaka OU, Tamaki EM. O papel da avaliação para a tomada de decisão na gestão de serviços de saúde. Ciênc Saúde Coletiva. 2012;17(4):821-4.

25. Coelho MO, Jorge MSB. Tecnologias das relações como dispositivo de atendimento humanizado na atenção básica à saúde na perspectivado acesso, do acolhimento e do vínculo. Ciênc Saúde Coletiva. 2009;14(Supl 1):1523-31.

26. Zancan L, Carvalho AI, Lobato MF, Rocha MR. Articulação intersetorial na gestão para a promoção da saúde. In: Gondim R, Grabois V, Mendes Junior WV, organizadores. Qualificação dos Gestores do SUS. $2^{\mathrm{a}}$ ed. Rio de Janeiro: Fiocruz/ENSP/EAD; 2011. p. $297-$ 310.

27. Castanheira ERL, Dalben I, Almeida MAS, Puttini RF, Patricio KP, Machado DF, et al. Avaliação da qualidade da Atenção Básica em 37 municípios do Centro - Oeste paulista: características da organização da assistência. Saúde Soc. 2009;18(2):84-8.

28. Silva AJM. Epidemiologia e planejamento e Saúde. In: Epidemiologia \& Saúde. Rouquayrol MZ, Silva MGC. Rio de Janeiro: Med Book, 2013. p 467-71.

29. Russo RFSM, Ruiz JM, Cunha RP. Liderança e influência na fase de gestão de projetos. Prod. 2005;15(3):362-75.
30. Corradi EM, Zgoda LTRW, Paul MFB. O gerenciamento de conflitos entre a equipe de enfermagem. Cogitare Enferm. 2008;13(2):184-93.

31. Sousa MHL, Souza RMP, Silva MGC. Alocação de Recursos na Saúde. In: Epidemiologia \& Saúde. Rouquayrol MZ, Silva MGC. Rio de Janeiro: Med Book; 2013. p 585-99.

32. Santos CF, Ariente M, Diniz MVC, Dovigo AA. $\mathrm{O}$ processo evolutivo entre as gerações $\mathrm{x}$, $\mathrm{y}$ e baby boomers. In: Anais do XIV SEMEAD Ensino e Pesquisa em Administração; 13 a 14 Out. 2011; Faculdade de Economia, Administração e Contabilidade da Universidade de São Paulo. São Paulo: Universidade de São Paulo; 2011. p. 1-14.

33. Barreto SO, Freitas LC, Del Prette ZAP. Habilidades sociais na comorbidade entre dificuldades de aprendizagem e problemas de comportamento: uma avaliação multimodal. Psico (Porto Alegre) 2011;42(4):503-10.

\section{Endereço para correspondência:}

Nayane Coelho Sales

Universidade Estadual do Ceará

Avenida Dr. Silas Munguba, 1700

Campus do Itaperi

CEP: 60740-000 - Fortaleza - CE - Brasil

E-mail: nayane.sales@gmail.com 